\title{
PEMFC stack diagnosis based on external magnetic field measurements
}

\author{
M. Le $\mathrm{Ny}^{1,2,3,4} \cdot$ O. Chadebec ${ }^{3,4}$ - G. Cauffet ${ }^{3,4}$. \\ S. Rosini ${ }^{5} \cdot$ Y. Bultel ${ }^{1,2}$
}

\begin{abstract}
A new diagnosis method based on the measurement of the magnetic field surrounding a PEMFC stack was developed. The method relies on the measurements of the magnetic field signature generated by the current distribution in the PEMFC. It is possible to estimate an averaged $2 \mathrm{D}$ current density identification by solving an inverse problem, based on the relationship between the currents and a magnetic field. In this work, 30 sensors fixed around the stack are used to perform the cartography within a second. This technique was implemented on a PEMFC stack consisting of 30 cells with a large active area of $220 \mathrm{~cm}^{2}$ and was capable to capture the current density change from a healthy state to a faulty one. Our approach is robust and allows us to distinguish between either membrane drying or electrode flooding by varying the air stoichiometry.
\end{abstract}

Keywords Current density identification - Fuel cell . Magnetostatics · Diagnosis

Y. Bultel

yann.bultel@lempi.grenoble-inp.fr

Univ. Grenoble Alpes, LEPMI, 38000 Grenoble, France

2 CNRS, LEPMI, 38000 Grenoble, France

3 Univ. Grenoble Alpes, G2ELab, 38000 Grenoble, France

4 CNRS, G2ELab, 38000 Grenoble, France

5 Univ. Grenoble Alpes, CEA, LITEN, 38054 Grenoble, France

\section{Introduction}

The proton exchange membrane fuel cell (PEMFC) is one of the most compelling energy conversion devices, particularly as a power source for stationary and mobile applications (stationary power generation, small portable applications, powering cars...). However, reliability, cost and durability appear to be the most important considerations in achieving successful commercialization of PEMFC (targets: 40,000 h for stationary applications and about $5000 \mathrm{~h}$ for automotive) [1]. On the one hand, electrode water flooding and membrane drying can induce reversible degradation impeding the PEMFC performances. On the other hand, PEMFC are prone to chemical, mechanical, or thermal hard constraints that inevitably lead to their degradation. These phenomena remain poorly understood but non-uniform fuel/air flows, hot spots and nonuniformity of materials within the stack may be reasons for such degradations. Nevertheless, all these degradation classes induce fuel cell voltage and performance decline and lifetime reduction.

The feasibility of PEMFC diagnosis by electrochemical impedance spectroscopy (EIS) [2], acoustic emission [3] and electrochemical noises [4] has been demonstrated. Measurements of cell voltages are the simplest to perform. They can detect voltage drops, indicating failures necessitating the immediate shutdown of the stack. So far, the EIS has been demonstrated to be a powerful, non-invasive, in situ, experimental diagnosis technique. Impedance measurements have mainly been used for fuel cell diagnosis to determine the internal resistance of such systems, which is partly related to membrane resistance. This resistance depends on water content and fuel cell temperature. Merida et al. [5] and Le Canut et al. [6] used EIS to detect membrane electrode assembly (MEA) drying or flooding. An 
increase in the impedance value was observed under flooding conditions compared to optimal conditions. Some authors [7, 8] coupled impedance spectroscopy with an equivalent electrical circuit model for EIS analysis to detect the onset of flooding. Lee et al. [9] used a similar model to estimate the state and lifespan of fuel cells in operation and observe degradation. Routine experimental methods based on different techniques devoted to the evaluation of cell state (Cell voltage, Acoustic emission, Electrochemical noises, EIS, etc.) are certainly powerful, but remain unsuitable when dealing with the heterogeneous aspects of the ageing phenomena taking place in the cell. Indeed, owing to the cell geometry, the uniformity of the chemical, physical, and electrical values is never perfectly achieved. Therefore, a technique allowing measurement of either membrane hydration state or electrode flooding, sensitive to localized phenomena would be of major interest in the development of a better water management strategy.

Beyond these non-invasive techniques, numerous experiments with in situ techniques have been developed to determine the current distribution over the cell surface [1015]. For example, printed circuit boards like the Current Scan Lin S++ device [14], inserted between two monopolar plates, can be used to map the current distribution in the stack with good resolution for a large surface area inside the stack. Among these internal current measurement techniques, it is worth mentioning the one based on the direct measurement of the internal magnetic field [15]. Non-invasive methods based on measurements of the magnetic field induced by current production within the stack have been proposed in literature for PEMFC cell [16] and stack $[17,18]$ diagnosis. The measurement of magnetic fields allows for the building of a three dimensional picture of the internal current density possible. As many phenomena such as MEA degradation, membrane drying and electrode flooding affect the current density distribution, magnetotomography can supply a lot of information about the operating conditions within a fuel cell. Moreover, since this method is non-invasive, it does not modify the current distribution within the stack. Previously magnetic field cartography has been performed using a 3-axis magnetic sensor displaced around the PEMFC stack via a robot. 300 measurements are necessary to obtain good resolution on current distribution mapping $[17,18]$. Such a system is extremely complex to develop and can only be used in a laboratory context. The originality of the approach presented here is that it requires a very small number of magnetic field measurements. The instrumentation is facilitated and the use of a robot moving a sensor is no longer necessary. The number of measurements is reduced down to 30 while maintaining an acceptable resolution. This allows us to fix 30 sensors on the stack and to perform the cartography within a very short delay (a second) against
15 min with the other methods. The major advantage is that the internal state of the stack does not vary during the measurement time. The low number of sensors is made possible thanks to assumptions on the current distribution and to an adequate choice of positioning and orientation according to the stack dimensions.

This technique was implemented on a real PEMFC stack. In order to compare the results with a reference, an internal current density measurement system, is introduced inside the stack. Even if this intrusive tool almost certainly disrupts the operation mode; it enables the comparison and validates our approach. Sensitivity to air stoichiometry was investigated in this work. By varying this parameter around nominal conditions, the influence of drying and flooding conditions on the current density distribution is then investigated based on this new diagnosis tool. Our approach was developed to be mainly sensitive to current density change when the mode of operation of the PEMFC stack is degrading from a healthy state to a faulty one.

\section{Evidence of the magnetic field change with current distribution heterogeneity}

The aim of this section is to exhibit the link between the faults occurring inside the fuel cell and the magnetic field generated. The first part of this section describes the equations of the direct model we used. Then the second part use this direct model in order to show that there exist a magnetic field correlated to these faults which we could make use for a diagnosis system.

\subsection{Description of the direct model}

A physics-based model of PEMFC stack or direct model was developed to simulate the 3D distribution of the current density, cell potential, streamlines distribution [19] and finally the induced magnetic field around the stack. The steady-state behaviour of a PEMFC stack was modelled to investigate on the one hand, the impact of changes in material conductivities, electrical configuration and on the other hand the effects of anomalies (contact resistances, etc.) on the electrical and magnetic characteristics. This model is composed of multiple single repeat units stacked on top of each other and sandwiched between two end plates along the z-axis. The stack consists of several cells and current collection was considered to be performed on the edge of the PEMFC stack. It is important to remember that there is only one region for the entire thickness of an MEA. The cathode, the anode and the membrane are not distinguished. All the physical phenomena that take place in these different layers are averaged over the MEA thickness and represented by the local polarization law. A 
model of the electrical interaction in a stack was used to simulate its electrical behaviour. PEMFC Stack models require the consideration of interaction between cells taking into account that single cells have different locations and cannot be identical from a material properties or operational conditions point of view. It provides a three-dimensional current density and potential distributions within the stack. The local performance of the individual cells must compete against the general voltage equations, which govern the current through the stack, if there are variations in cell performance, or changes in operating conditions from cell to cell. Modelling the electrical behaviour of the stack is performed by solving the steady state charge conservation equation and the local Ohm's law:

$\operatorname{div} \mathbf{j}$

$\mathbf{j}=\sigma \mathbf{E}$

$\mathbf{E}=\operatorname{grad} V$

where $\mathbf{j}$ is the current density, $V$ the potential and $\sigma$ the conductivity.

Combining these three equations leads to the following transport equation:

$\operatorname{div} \sigma \operatorname{grad} V=0$

This latter equation (Eq. (4)) is used to describe charge transport in some regions of the stack where no electrochemical processes take place (i.e., bipolar plate, current collector and end plate). In the MEA, electrochemical phenomena induce a potential jump and an additional term related to the electrochemical behaviour of the MEA needs to be added [19]. So, Eq. (3) has been modified to include an additional term related to this electromotive field $\mathbf{E}_{\mathbf{m}}$. So the electric field can be written in the following form:

$\mathbf{E}=\mathbf{E}_{\mathbf{m}}-\operatorname{grad} V$

where $\mathbf{E}_{\mathbf{m}}$ is the voltage drop induced by the MEA divided by MEA thickness. This term allows the modelling of a force that can drive charges through a medium. In stacks this force arises because of the electrochemical phenomena occurring inside the MEA. Thanks to this known source term, the electrical behaviour of a stack can be easily modelled with a set of two distributions parameters: the conductivity and the electromotive field. Thus, the transport equation becomes:

$\operatorname{div} \sigma \operatorname{grad} V=\operatorname{div} \sigma \mathbf{E}_{\mathbf{m}}$

It is worth mentioning that the electromotive field is null inside bipolar plates and end plates. Its value in the MEA is the open circuit voltage (OCV) divided by the thickness of the MEA in the linear case.

The electrochemical response of the MEA is given by an empirical polarisation curve:
$\Delta \mathrm{V}(j)=U_{O C V}-R_{M E A} j-A \ln \left(\frac{j}{j_{e q}}+1\right)$

where $U_{O C V}$ is the OCV of one cell, $R_{M E A}$ the total resistance of the MEA and $A$ and $j_{e q}$ are fitted parameters that represent the activation overpotential due to electrochemical reactions.

This kind of equation is very efficiently solved numerically by the finite volume method (FVM). FVM involves meshing a domain and then integrating the partial differential equation over each small volume. A NewtonRaphson algorithm was used to couple this equation with Eq. (6) in MEA regions. The procedure is presented in Le $\mathrm{Ny}$ et al. [19].

Table 1 gives the parameters values used for each region in the case of a healthy fuel cell stack. Parameters used for a MEA for non-linear polarization law are summarised in the Table 2.

The magnetic field distribution around the stack is then calculated from the current distribution thanks to the Biot and Savart law:

$\mathbf{B}(\mathbf{r})=\frac{\mu_{0}}{4 \pi} \iiint_{\Omega} \frac{\mathbf{j}\left(\mathbf{r}_{\Omega}\right) \times\left(\mathbf{r}-\mathbf{r}_{\Omega}\right)}{\left|\mathbf{r}-\mathbf{r}_{\Omega}\right|^{3}} d \Omega$

where $\mathbf{B}$ is the magnetic induction at point $\mathbf{r}, \mathbf{j}$ is the current density at the integration point $\mathbf{r}_{\mathbf{\Omega}}, \mu_{O}$ is the permeability of free space and $\Omega$ is the volume of the stack where current flows.

Please note that the Biot and Savart law is only valid for static current sources and when there is no ferromagnetic parts in the close environment. In case this would not be the case, the static Maxwell equations would have to be solved by using more complex numerical methods (like Finite Element Methods for example). But it would not drastically modify the methodology presented in this article.

Table 1 Stack parameters

\begin{tabular}{ll}
\hline Current collector conductivity & $5 \times 10^{7} \mathrm{~S} \mathrm{~m}^{-1}$ \\
End plate conductivity & $5 \times 10^{7} \mathrm{~S} \mathrm{~m}^{-1}$ \\
Bipolar plate conductivity & $5 \times 10^{3} \mathrm{~S} \mathrm{~m}^{-1}$ \\
Current collector thickness & $1 \mathrm{~mm}$ \\
End plate thickness & $2.5 \mathrm{~mm}$ \\
Bipolar plate thickness & $4 \mathrm{~mm}$ \\
MEA thickness & $0.1 \mathrm{~mm}$ \\
Current collector area & $0.02 \times 0.02 \mathrm{~m}^{2}$ \\
End plate area & $0.14 \times 0.14 \mathrm{~m}^{2}$ \\
Bipolar plate surface area & $0.14 \times 0.14 \mathrm{~m}^{2}$ \\
MEA surface area & $0.1 \times 0.1 \mathrm{~m}^{2}$ \\
\hline
\end{tabular}


Table 2 Parameters used for a MEA for linear polarization law (healthy PEMFC stack)

\begin{tabular}{ll}
\hline MEA electromotive force $\left(U_{O C V}\right)$ & $1 \mathrm{~V}$ \\
MEA resistance per unit area $\left(R_{M E A}\right)$ & $1.79 \times 10^{-5} \Omega \mathrm{m}^{2}$ \\
MEA non-linear parameter $(A)$ & $0.025 \mathrm{~V}$ \\
MEA non-linear parameter $\left(j_{e q}\right)$ & $90 \mathrm{~A} \mathrm{~m}^{-2}$ \\
\hline
\end{tabular}

Table 3 Nominal operation conditions of the PEMFC stack GENEPAC

\begin{tabular}{ll}
\hline Current & $110 \mathrm{~A}$ \\
Pressure & $1.5 \mathrm{bar}$ \\
Relative humidity (Air) & $50 \%$ \\
Relative humidity (H2) & $50 \%$ \\
Stoichiometry (Air) & 2 \\
Stoichiometry (H2) & 1.5 \\
Temperature & $80{ }^{\circ} \mathrm{C}$ \\
\hline
\end{tabular}

\subsection{Influence of the current density distribution on the magnetic field}

As part of our study, the magnetic model is used on current distributions generated by the electrical model presented in the previous section of this document. The main advantage of this direct model is to generate current distribution in the stack representative of actual conditions. This direct model is able to provide the current distribution inside the stack and the induced magnetic field around the stack. For example, Fig. 1 shows the simulated current density considering a healthy stack (i.e., without anomalies) and a faulty stack (i.e., with an anomaly on one corner of the 4th cell) and magnetic field on a square path located on the middle section of the stack.

In both simulations, the magnetic field is computed on a few points around the stack as shown in Fig. 1. Figure 2 presents the magnitude of the magnetic field for these healthy and faulty stacks. The magnetic field in the presence of a fault is substantially equal to the magnetic field generated by a healthy cell with a maximal value of about $125 \mu \mathrm{T}$ (Fig. 2). The anomaly on the corner of the 4th cell creates a relative variation of the magnetic field that is very low: about $7 \mu \mathrm{T}$, or $5 \%$ of the total field as presented on Fig. 2. This is only one illustrative example but other simulations showed the similar phenomena: in general, the amplitude of the variation due to the anomaly is always much lower than the amplitude of the normal operation of the stack. The maximum "healthy magnetic field" we can get is around $600 \mu \mathrm{T}$ while the maximum amplitude due to the faults is around $30 \mu \mathrm{T}$.
Therefore, we have two antagonistic constraints for our diagnostic system. First, the magnetic sensors must have a high magnetic range in order to support (without saturation) the intense magnetic field related to the normal operation of the stack. Secondly, at the same time, the sensors must also be accurate enough in order to detect the weak magnetic fields variations generated by the faults inside the stack. These two constraints are antagonist for a measurement system because the first one requires a low sensitivity while the other requires a high sensitivity. But an innovative technique for solving this dilemma will be shown later in this paper. Until then, we can already state that our direct model has demonstrated the link between the faults (current density variations) and the external magnetic field. We could then make use of this magnetic field in order to design a diagnosis system that would detect the faults inside the stack. The big advantage with the external fields is that the magnetic field measurement doesn't require to instrument the interior of the stack. Then, such diagnosis system is non-invasive, which is an advantage over other invasive diagnosis systems.

\section{Design of the current density measurement system}

In this section, we present the methodology we used in order to design a non-invasive measurement tool that would estimates the current density inside the stack from the measurement of the external magnetic field. This current density measurement system could be then used to design a complete diagnosis tool for fuel cell in a future work.

Estimating the current density from the external magnetic field is not a straightforward task. In fact, it requires to inverse the Biot and Savart law (Eq. 7). Such inverse problem is ill posed, i.e., it is very sensitive to noise and as such, it cannot be solved with classic optimization approach. It requires the use of regularization techniques in order to filter out the noise attached to all the measurements. These considerations about ill posed inverse problem have been more detailed in [20]. In this section, we will only briefly describe how we have diminished the overall noise of our measurement system and how we accelerated the measurement process thanks to an innovative arrangement of our magnetic sensor around the stack.

\subsection{Strategy for noise reduction}

In the previous section, we already noticed that the faults (current inhomogeneities) create a low magnetic field variation (around $30 \mu \mathrm{T}$ ) compared to the intense magnetic 
Fig. 1 Current density distribution $\left(\mathrm{A} \mathrm{cm}^{-2}\right)$ and magnetic field around the stack. Upper: Healthy stack with a homogeneous current density distribution. Lower: Faulty stack (with a resistive default on the 4th cell) with a heterogeneous current density distribution
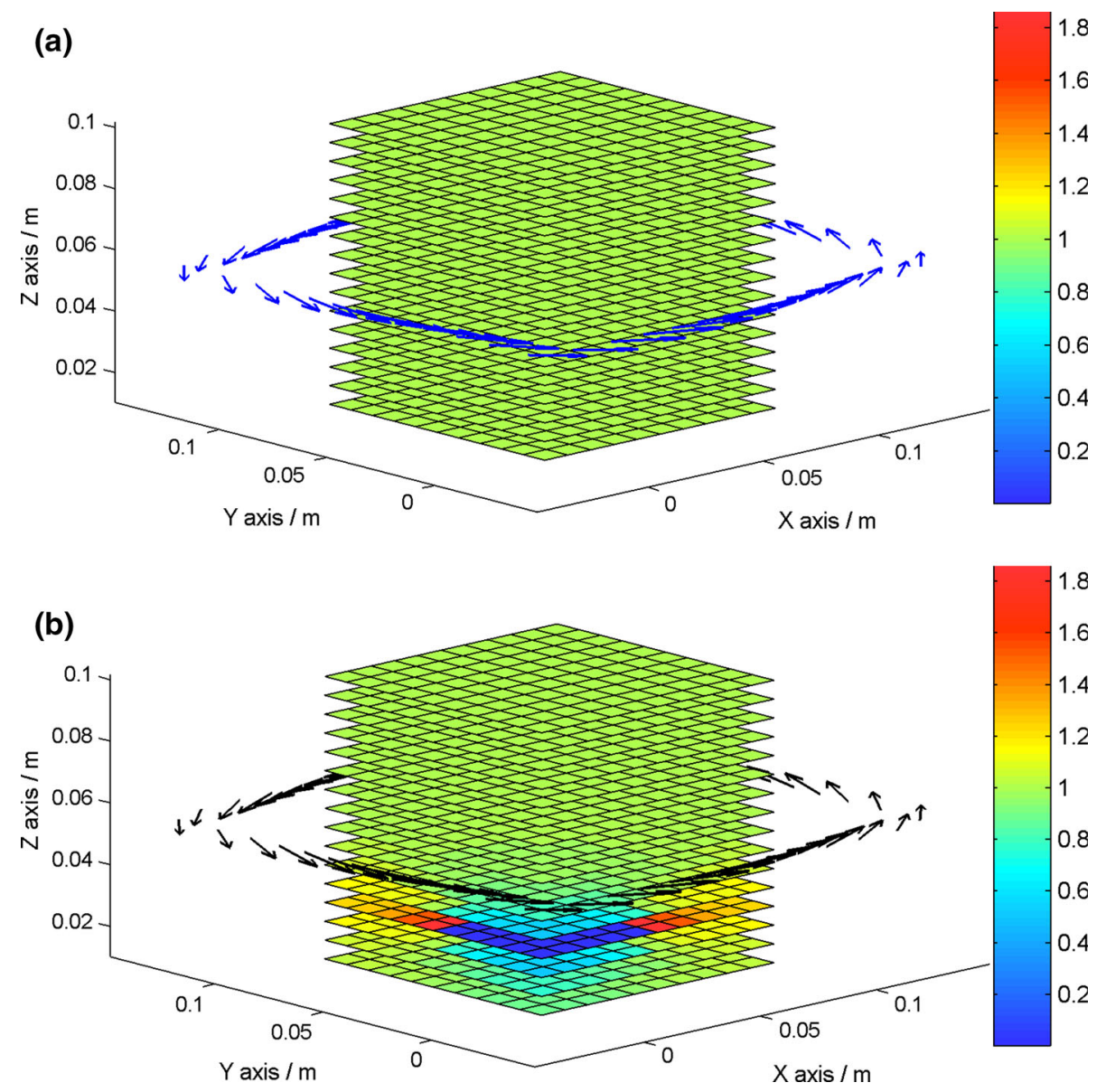

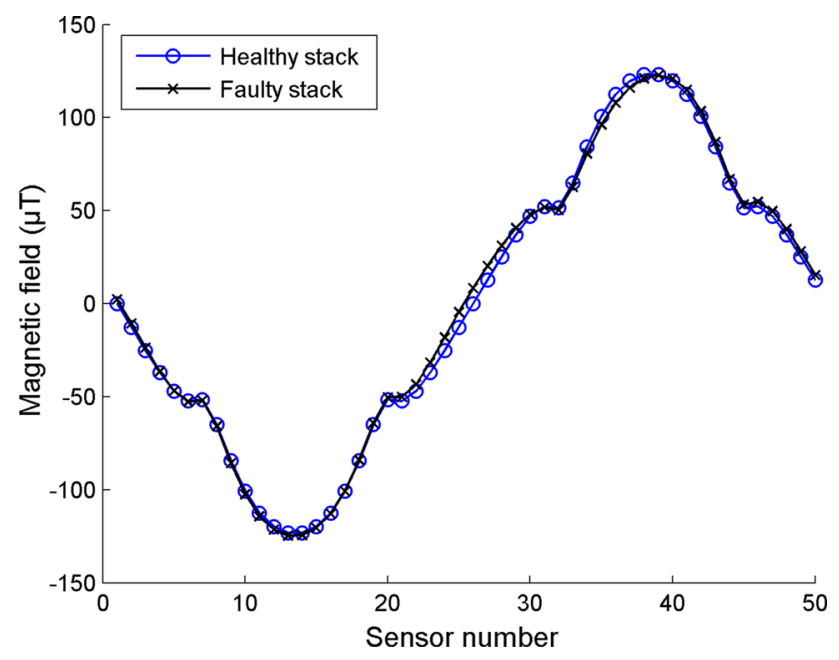

Fig. 2 Magnitude of the magnetic field for a healthy and a faulty stack with a resistive default on the 4 th cell

field of a healthy stack (around $600 \mu \mathrm{T}$ ). Consequently, we have to choose the range of our magnetic sensor such a way it can always support $600 \mu \mathrm{T}$ without saturating. Also it has to support other perturbations like the magnetic field of earth $(50 \mu \mathrm{T})$ plus some security margin $(150 \mu \mathrm{T}$ for a first design step). This lead to a high magnetic range of $800 \mu \mathrm{T}$ that implies a low sensor sensitivity. But the low magnetic field variations that are of interest for us requires a high accuracy, i.e., a high sensitivity.

We circumvented this problem by noticing that the intense magnetic field is only correlated to the total current flowing through the stack. This total current can be measured with a classic current meter (amp meter) without involving magnetic field measurements. As a result, we do not need to measure the magnetic field that is related to the total current, i.e., that this intense field is of no interest for us. This intense and useless field has the characteristic to be quasi ortho-radial. Therefore, if we only measure the magnetic component that is perpendicular to this field (i.e., the components that are quasi-radial and quasi-axial), the magnetic sensors will never saturate because of this intense field. Doing so allow us to use magnetic sensors with a range divided by four, i.e., with a range around $200 \mu \mathrm{T}$. Thanks to these more sensitive sensors, we can estimate more accurately the low magnetic field variations due to the current inhomogeneities which are themselves due to the faults occurring inside the stack. Therefore, thanks to this technique, we were able to multiply by four our 
robustness against the noises of the measurement system. The disadvantage of this technique is that we have less information available because we can now only measure two components of the magnetic field instead of the three components available. But the increase of the sensitivity compensates much more this loss of information.

Also, it is worth mentioning that on a real stack, the positioning of sensors is not fully accurate (due to the tolerances). Consequently, the total current mode still creates some magnetic field residue on the sensor array, which is difficult to predict. Therefore, this unpredictable residual field acts as noise. A good way to cancel this noise is to keep the total current constant over time and to make differential measurements over time.

\subsection{Magnetic sensor arrangement around the stack}

In the current state of art, the external magnetic field is scanned with the help of a robot that moves a magnetic sensor around the stack $[17,18]$. This technique allows the acquisition of 300 hundreds magnetic field measures that are useful for determining the current density inside the stack. But this acquisition last around $15 \mathrm{~min}$. During this time, the internal state of the fuel cell can vary and lead to wrong current estimation.

In order to circumvent this problem, we decided to make only 30 measures. Thanks to this low number of measures, we are not forced anymore to use a robot that moves only one sensor. Instead, we use 30 sensors that are fixed around the stack. This allow us to measure the magnetic field within a second. The major advantage is that the internal state of the stack does not (almost) vary during the quick measurement time. Also, the internal state can be followed over time.

Of course, using less measures (30 against 300) has a drawback: less information is obtained and the spatial resolution is degraded. Nevertheless, there were some redundancy of information within the previous hundreds of measures. Also, the regularization techniques that filter the noise destroys as well a good amount of the useful information. In the end, it has been shown that even if the use of few (30) measures degrades a bit the spatial resolution, it remains still acceptable [20]. In our opinion, the higher speed acquisition exceeds the drawbacks (lower spatial resolution).

Figure 3 shows the final arrangement of our magnetic sensors around the stack. In this first design step, only the quasi-radial component of the field is measured (represented by red arrows on Fig. 3). Also, the magnetic field is only measured on a square path located on the middle section of the device (Fig. 3). In order to maximize the signal to noise ratio, sensors are located as close as possible

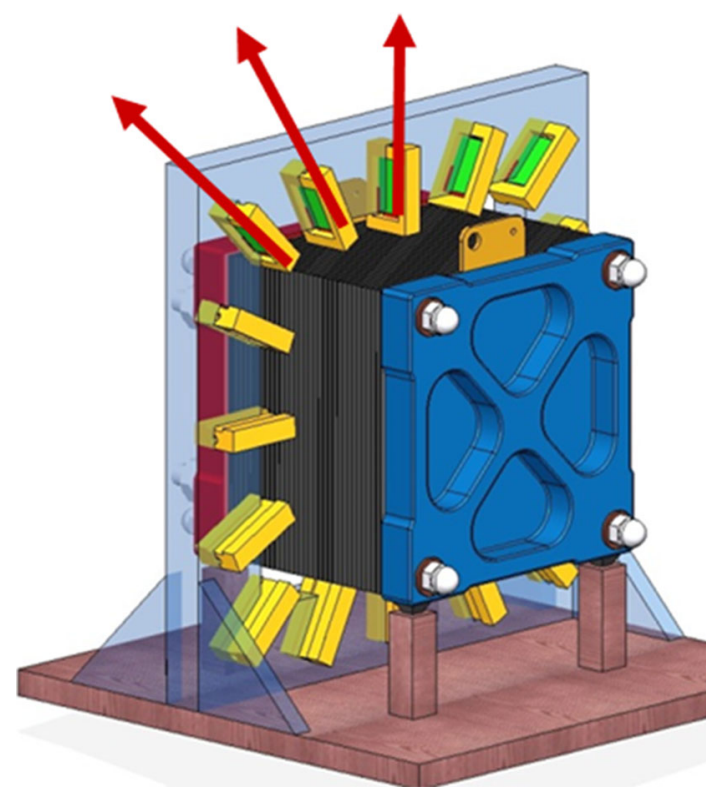

Fig. 3 Schematic representation of the stack (blue, red and black) and orientation of single-axis fluxgate magnetic sensors (red solid arrows). (Color figure online)

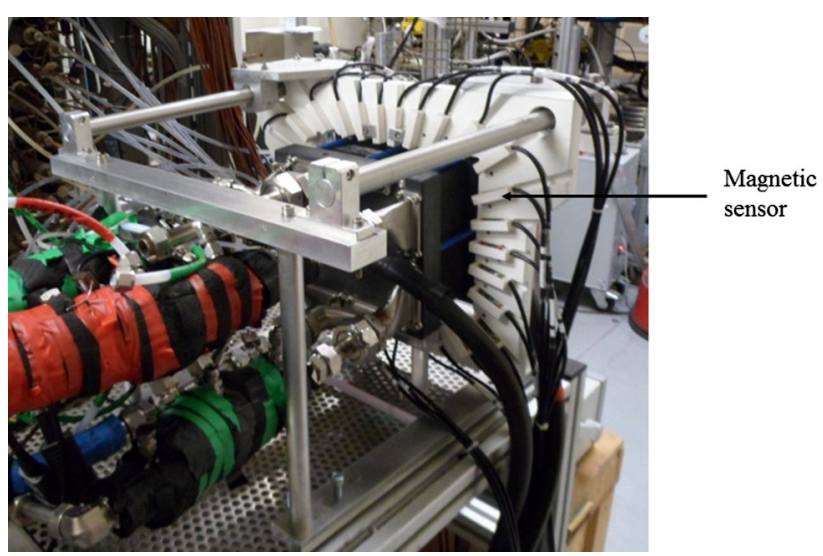

Fig. 4 Photo of the PEMFC stack GENEPAC

to the stack. For a future work, we could imagine to also measure the quasi axial component as well as to measure the magnetic field all around the stack. But for this first design step, we limited ourselves to what is pictured on Fig. 3. This lead to the fact that we do not estimate right now a full 3D cartography of the current density. What we get is a (weighted) average of the 2D current density cartography in the section of the stack. More specifically, the section in the middle of the stack (under the sensors) has the highest weight in the average of the $2 \mathrm{D}$ current distribution.

This methodology was previously validated on a stack simulator in order to check the feasibility of the system [20]. 


\subsection{Description of the experimental set-up}

We applied our new measurement system on a stack that consists of 30 cells with an active area of $220 \mathrm{~cm}^{2}$, connected in series from the GENEPAC technologies. Each cell is composed of one MEA where the electrochemical reactions take place and one bipolar plate with serpentine flow field. This latter is a multifunctional component which ensures proper reactive gases distribution in the cell and evacuation from the cell, separates the different cells of the stack, collects the current away from the cell, assists the heat and water management, and ensures the mechanical stability and sealing of the stack. Table 3 summarises the nominal operational conditions.

Figure 4 presents the PEMFC stack on the test bench including the external magnetic field measurement system. The 30 magnetic sensors are fixed on a single plane around the GENEPAC stack and enable an instantaneous measurement. The technology used here for the magnetic sensors is the fluxgate technology (the FLCXS2 reference from Stefan Mayer Instruments).

A printed circuit board or $\mathrm{S}++{ }^{\circledR}$ card [14] inserted between the 15th and 16th cells allows measurement of the in-plane current density distribution at different operating conditions. The $\mathrm{S}++{ }^{\circledR}$ card consists of a gold-platted segmented plate where each one of the $24 \times 20$ segments is a current sensor. The rather homogeneous distribution of current density recorded with the $\mathrm{S}++$ card is shown on Fig. 5 for a PEMFC stack working under nominal conditions (Table 1). The arrows display the location of the air inlet and air outlet (The exact design of gas flow fields is not presented for reasons of confidentiality). This invasive method is used to validate our approach.

\section{Experimental results}

The experiments described in this section are performed to predict the change in current distribution through the stack when anomalies are induced by changing operation conditions. In this study, inhomogeneous distribution of the current can be observed by varying the air stoichiometry and air hygrometry around the nominal conditions.

\subsection{Oxygen starvation}

The Air stoichiometry was first decreased from 2 down to 1.15 leading to oxygen starvation at the cathode. During all experiment, Stack is under Galvanostatic mode and current is equal to $110 \mathrm{~A}$. As shown in Fig. 6, we observed a change of $15 \mu \mathrm{T}$ in the magnetic field when lowering the Air stoichiometry. This value is within the range of the simulated magnetic field change (see Sect. 2). The magnetic field is

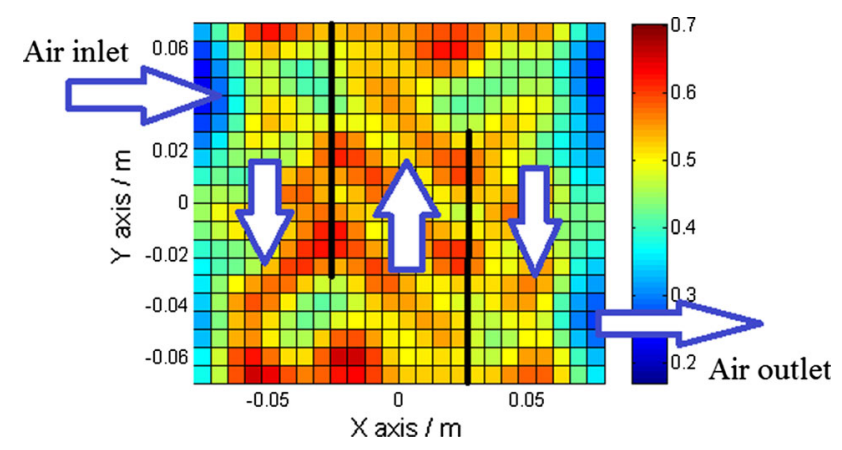

Fig. 5 Current density distribution $\left(\mathrm{A} \mathrm{cm}^{-2}\right)$ recorded by the $\mathrm{S}++$ card

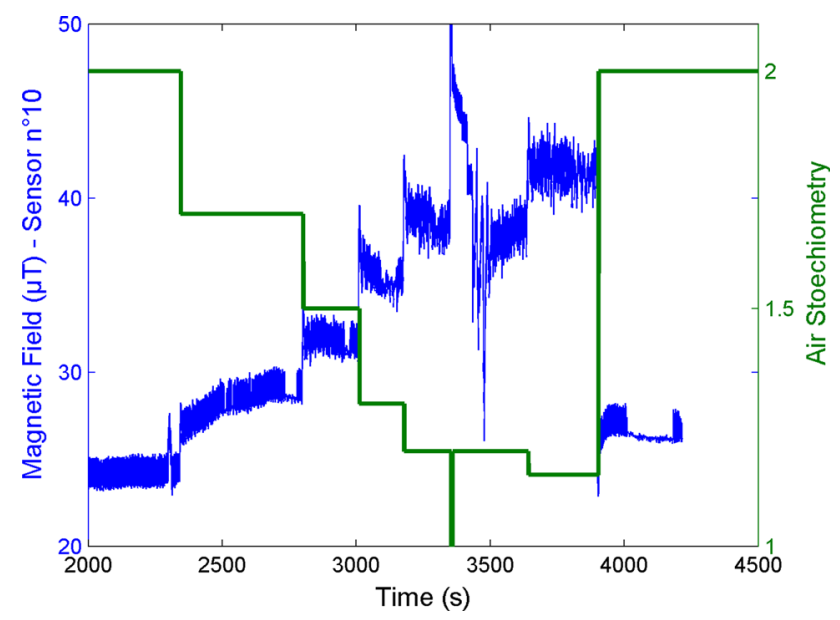

Fig. 6 Variations in the air stoichiometry and corresponding response of the magnetic field on a sensor

particularly sensitive to such variations and thus the current distribution.

Throughout the experiment, the magnetic field is recorded at $t_{0}$ when the PEMFC Stack is working under nominal condition and at different step $t_{i}$ for an air stoichiometry ranging from 1.7 down to 1.15 . The measured values of the magnetic field are averaged over approximately 160,000 points for an integration time of about $20 \mathrm{~s}$. This average value is used to reduce the noise level measurements. In order to obtain the change of magnetic field between the times $t_{0}$ and $t_{i}$, we then subtract these measurements as follows:

$\Delta \hat{B}=\hat{B}\left(t_{i}\right)-\hat{B}\left(t_{0}\right)$

The inverse problem allows the calculation of the variation of the current density from $t_{0}$ to $t_{i}$ :

$\Delta j=j\left(t_{i}\right)-j\left(t_{0}\right)$

It is worth mentioning that our reconstruction methodology [20] is sensitive only to the change in the current distribution heterogeneity with time between $t_{0}$ and $t_{i}$. 

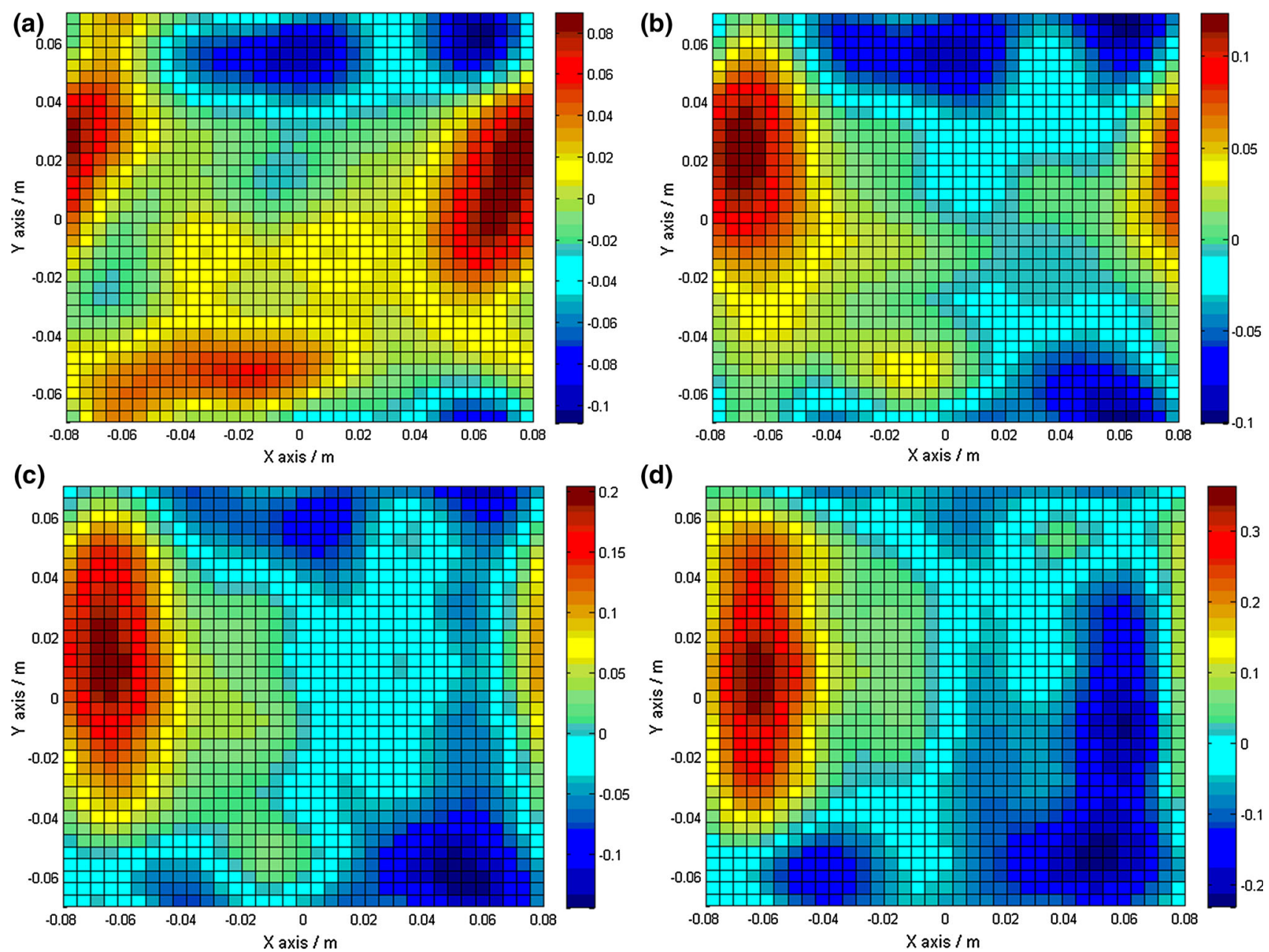

Fig. 7 Reconstructed current density change from magnetic field measurement for air stoichiometry ranging from 2 down to 1.7 (a), 1.5 (b), 1.3 (c) and $1.15(\mathbf{d})$
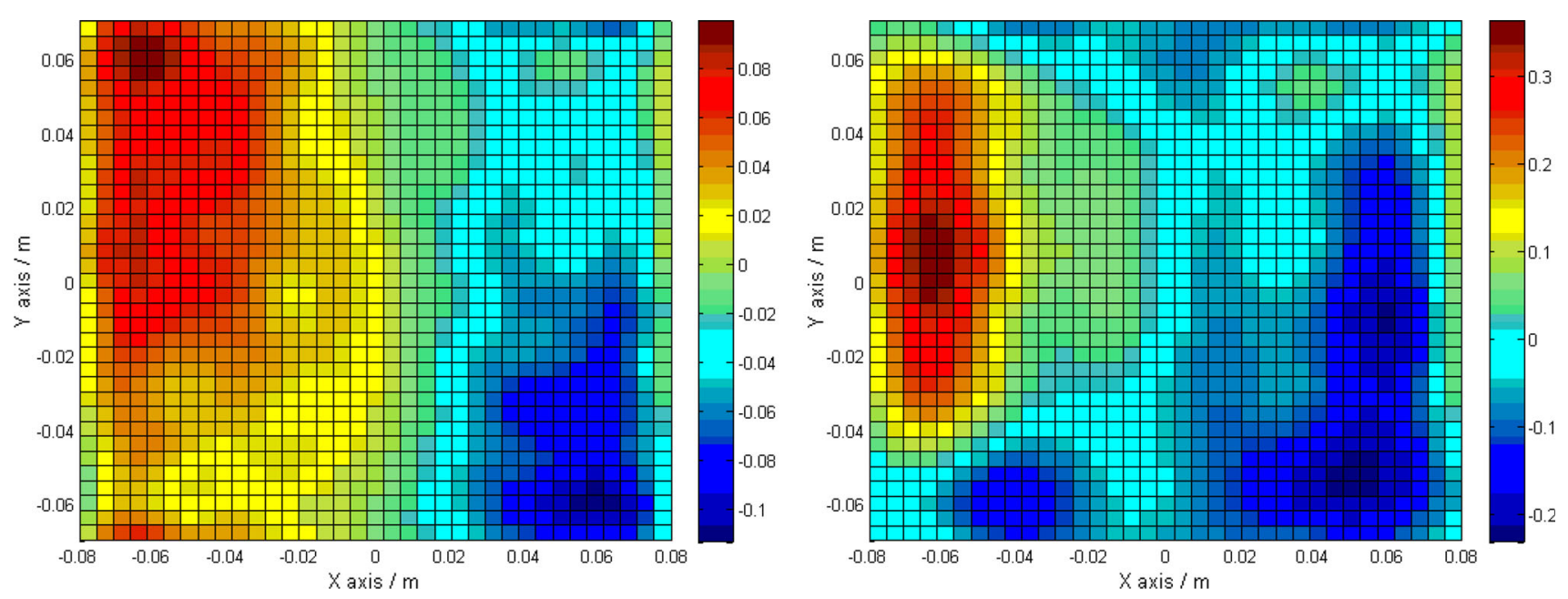

Fig. 8 Current density changes $\left(\mathrm{A} \mathrm{cm}^{-2}\right)$ from $\mathrm{S}++$ card $($ left $)$ and reconstructed current density change from magnetic field measurement (right) for air stoichiometry ranging from 2 down to 1.15 


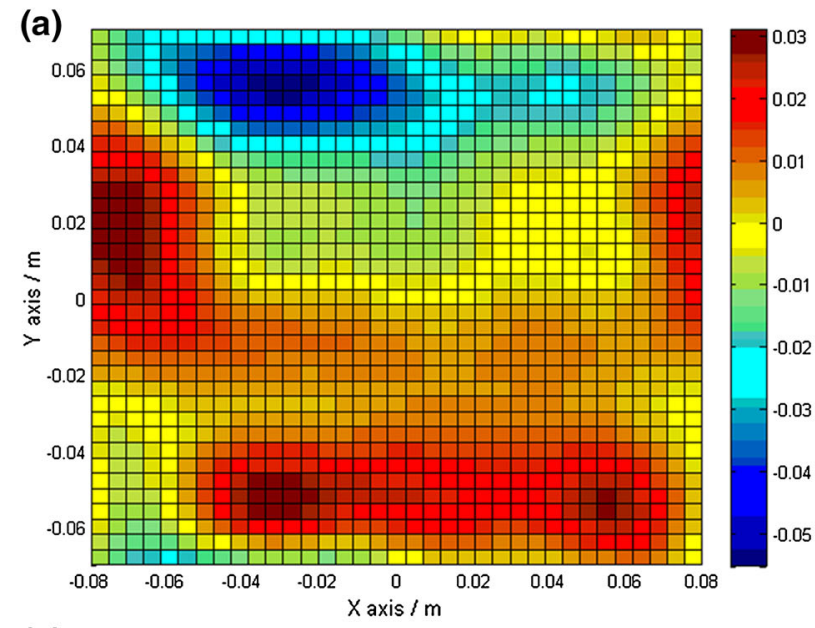

(c)

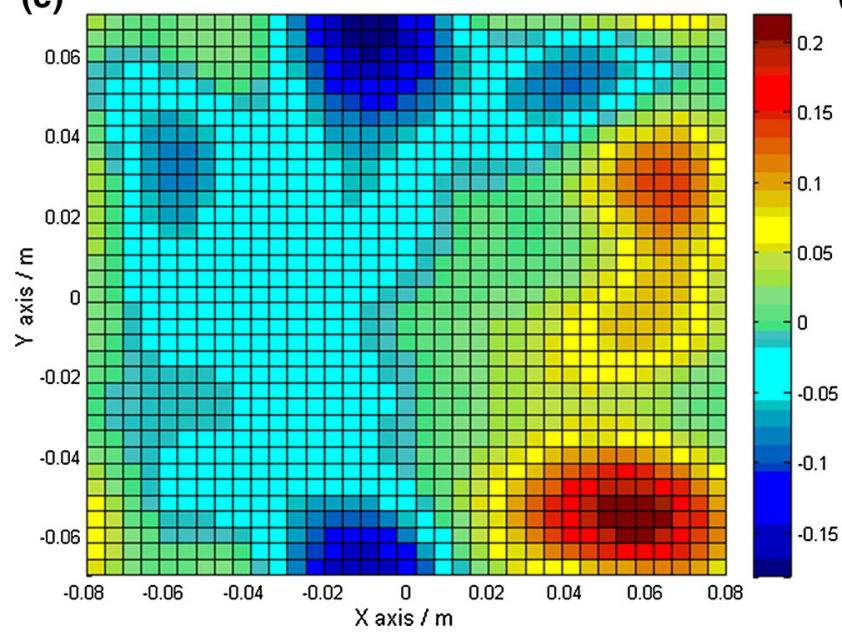

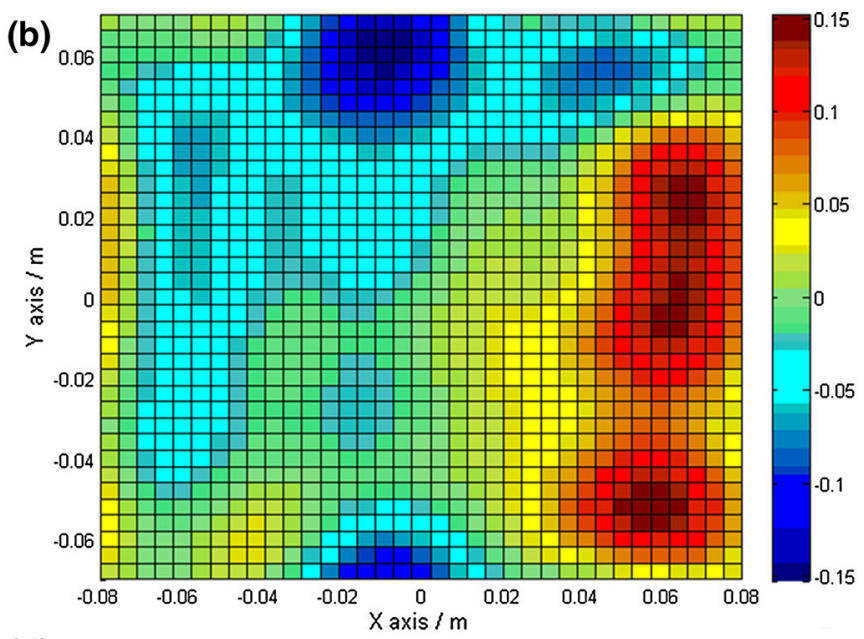

(d)

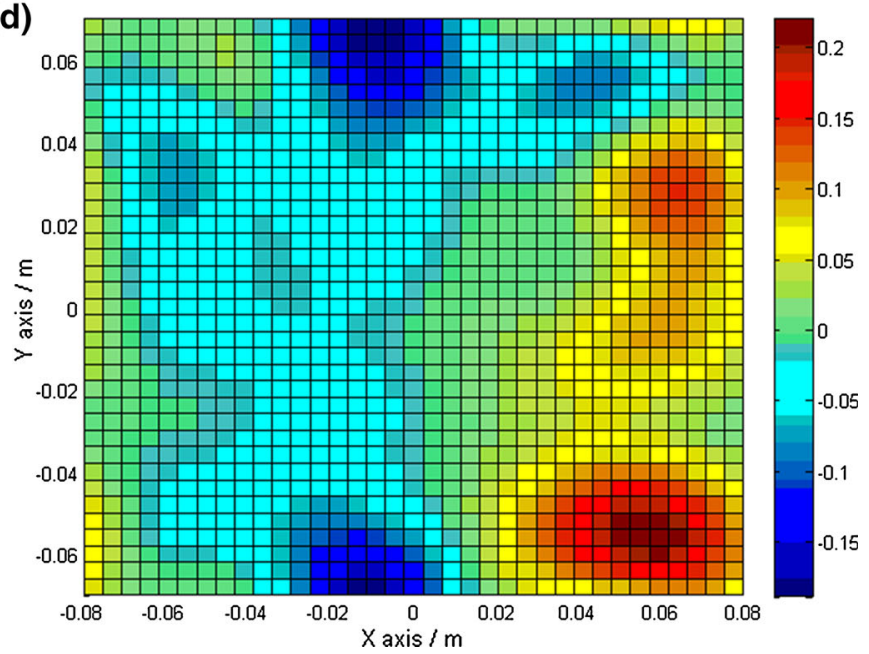

Fig. 9 Reconstructed current density change from magnetic field measurement for air stoichiometry ranging from 2 to 2.2 (a), 3 (b), 4 (c) and 4.5 (d)

Figure 7 displays the current density changes when the air stoichiometry decreases from 2 to 1.15 . The current density change remains rather small $\left(|\Delta j|<0.1 \mathrm{~A} \mathrm{~cm}^{-2}\right)$ when the air stoichiometry varies from 2 down to 1.7 for a mean current density equal to $0.5 \mathrm{~A} \mathrm{~cm}^{-2}$. Conversely, current density change becomes larger $(|\Delta j|>$ $0.25 \mathrm{~A} \mathrm{~cm}^{-2}$ ) when the air stoichiometry varies from 2 down to 1.15. Larger current density are observed closed to the air inlet meanwhile lower current density are obtained close to the gas outlet, conforming to oxygen depletion along the gas channel decreasing air stoichiometry. The effect is clearly related to oxygen starvation when the PEMFC is working at very low air stoichiometry.

In order to validate our non-invasive approach, these results were compared to those recorded by the $\mathrm{S}++$ card, even if this intrusive tool almost certainly partly disrupts the PEMFC operation. Figure 8 presents, the current density changes when the air stoichiometry is dropped from 2 down to 1.15 . Current density distribution is very heterogeneous when the PEMFC cell is working at low air stoichiometry in comparison to nominal conditions. Conforming to these current density distributions, larger current density changes are observed close to the gas inlet and outlet. From a general point of view, quite good concordance is observed when comparing both techniques even if some discrepancies can be detected. These differences are explained in part by the uncertainties induced by both methods. Another reason is that these tools do not measure the current at exactly the same locations. The $\mathrm{S}++$ card measures the current density on the bipolar plate surface, while our magnetic field tool determines the "mean" current densities across the entire MEA of the stack.

\subsection{Membrane dehydration}

In the second step, the air stoichiometry was increased from 2 up to 4.5 leading to membrane dehydration. Figure 9 displays the current density changes when the air 

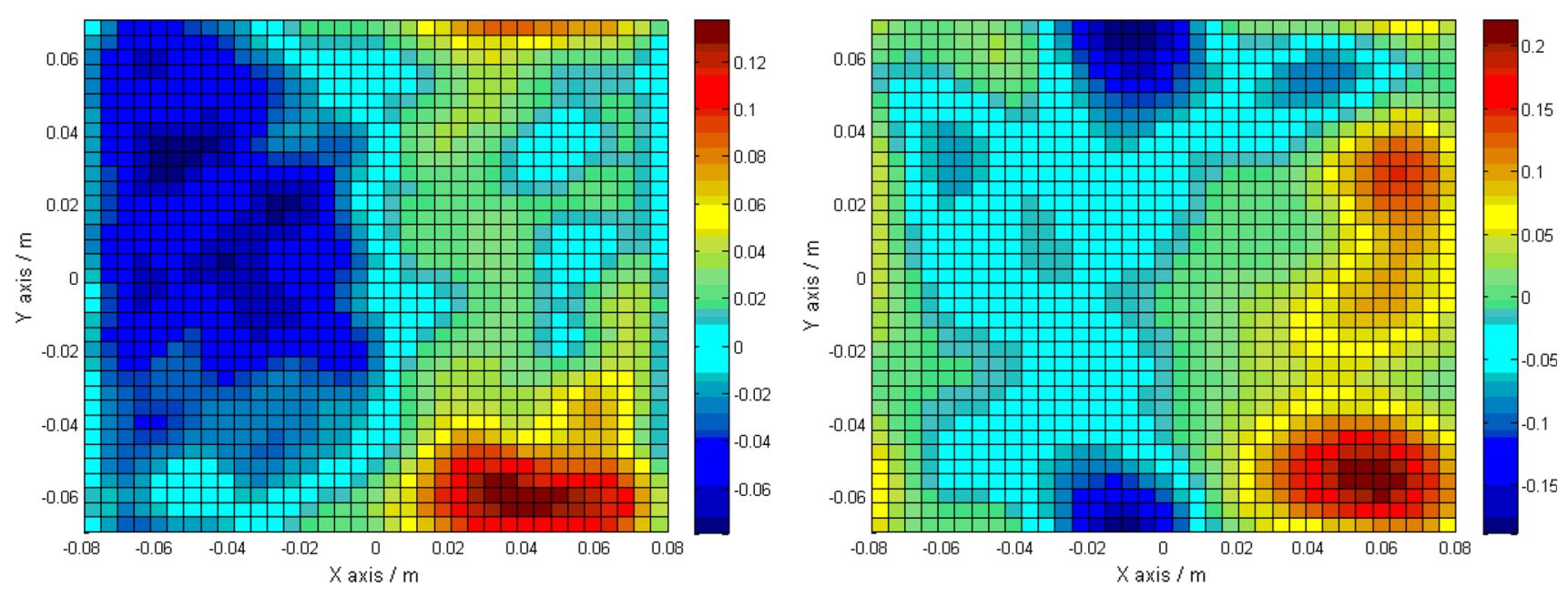

Fig. 10 Current density changes $\left(\mathrm{A} \mathrm{cm}^{-2}\right)$ from $\mathrm{S}++$ card $(l e f t)$ and reconstructed current density change from magnetic field measurement (right) for air stoichiometry ranging from 2 to 4.5

stoichiometry increases from 2 to 4.5 . The current density change remains rather small $(|\Delta j|<0.1)$ when the air stoichiometry varies from 2 to 2.2 for a mean current density equal to $0.5 \mathrm{~A} \mathrm{~cm}^{-2}$. Conversely, current density change becomes larger $(|\Delta j|>0.2)$ when the air stoichiometry varies from 2 up to 4.5 . Lower current density is observed close to the air inlet meanwhile larger current density is obtained close to the gas outlet, conforming to membrane dehydration along the gas channel increasing the air stoichiometry. The effect is clearly related to the high water removal process at the inlet part of the cell and hydration of the membrane along the channel because of water production by an oxygen reduction reaction at the cathode. Similar results are observed when the air hygrometry is decreased from 50 to $25 \%$.

Similarly to the previous operation conditions, these results were compared to those recorded by the $\mathrm{S}++$ card, in order to validate our non-invasive approach. Figure 10 presents, the current density changes when the air stoichiometry is increased from 2 up to 4.5. Current density distribution is very heterogeneous when the PEMFC cell is working at high air stoichiometry in comparison to nominal conditions. Larger current density changes are also observed close to the gas inlet and outlet. From a general point of view, a quite good agreement is observed when comparing both techniques even if some discrepancies can be detected.

\section{Conclusions}

In this study, a new and innovative magnetic tomography approach was proposed in order to reconstruct faults in PEMFC stacks. A sensor array was developed and designed to be sensitive only to current heterogeneities. This technique allows the use of a few magnetic sensors that have a low magnetic range of measurements but a high sensitivity and high dynamic.

The sensitivity of our system has been demonstrated for air stoichiometry change under nominal conditions. The sensitivity to air hygrometry was also observed. Our inversion algorithm is able to reconstruct current density distribution. In effect, the current density maps obtained with our magnetic tool showed good concordance with those obtained from the invasive $\mathrm{S}++$ card.

Even if the method has been validated on several test cases and has shown its potential, improvement of resolution with better inversion algorithms and better sensor array design remains necessary. This technique requires some improvement in order to correlate magnetic signatures and internal failures and to take advantage of the large bandwidth of the system.

\section{References}

1. Yousfi-Steiner N, Moçotéguy P, Candusso D, Hissel D, Hernandez A, Aslanides A (2008) A review on PEM voltage degradation associated with water management: impacts, influent factors and characterization. J Power Sources 183:260-274. doi:10.1016/j.jpowsour.2008.04.037

2. Fouquet N, Doulet C, Nouillant C, Dauphin-Tanguy G, OuldBouamama B (2006) Model based PEM fuel cell state-of-health monitoring via ac impedance measurements. J Power Sources 159:905-913. doi:10.1016/j.jpowsour.2005.11.035

3. Legros B, Thivel PX, Bultel Y, Boinet M, Nogueira RP (2010) Acoustic emission: towards a real-time diagnosis technique for proton exchange membrane fuel cell operation. J Power Sources 195:8124-8133. doi:10.1016/j.jpowsour.2010.07.045

4. Legros B, Thivel PX, Bultel Y, Nogueira RP (2011) First results on PEMFC diagnosis by electrochemical noise. Electrochem Commun 13:1514-1516. doi:10.1016/j.elecom.2011.10.007 
5. Merida W, Harrington DA, Le Canut JM, McLean GJ (2006) Characterisation of proton exchange membrane fuel cell (PEMFC) failures via electrochemical impedance spectroscopy. J Power Sources 161:264-274. doi:10.1016/j.jpowsour.2006.03.067

6. Le Canut JM, Abouatallah RM, Harrington DA (2006) Detection of membrane drying, fuel cell flooding, and anode catalyst poisoning on PEMFC stacks by electrochemical impedance spectroscopy. J Electrochem Soc 153:A857-A864. doi:10.1149/1. 2179200

7. Rodat S, Sailler S, Druart F, Thivel PX, Bultel Y, Ozil P (2010) EIS measurements in the diagnosis of the environment within a PEMFC stack. J Appl Electrochem 40:911-920. doi:10.1007/ s10800-009-9969-0

8. Tant S, Rosini S, Thivel PX, Druart F, Rakotondrainibe A, Geneston T, Bultel Y (2014) An algorithm for diagnosis of proton exchange membrane fuel cells by electrochemical impedance spectroscopy. Electrochim Acta 135:368-379. doi:10.1016/j. electacta.2014.04.108

9. Lee JH, Lee JH, Choi W, Park KW, Sun HY, Oh JH (2010) Development of a method to estimate the lifespan of proton exchange membrane fuel cell using electrochemical impedance spectroscopy. J Power Sources 195:6001-6007. doi:10.1016/j. jpowsour.2010.02.054

10. Stumper J, Campbell SA, Wilkinson DP, Johnson MC, Davis M (1998) In-situ methods for the determination of current distributions in PEM fuel cells. Electrochim Acta 43:3773-3783. doi:10.1016/S0013-4686(98)00137-6

11. Freunberger SA, Schneider IA, Sui PC, Wokaun A, Djilali N, Buchi FN (2008) Cell interaction phenomena in polymer electrolyte fuel cell stacks. J Electrochem Soc 155(7):B704-B714. doi:10.1149/1.2913095

12. Schulze M, Gulzow E, Schonbauer S, Knori T, Reissner R (2007) Segmented cells as tool for development of fuel cells and error prevention/prediagnostic in fuel cell stacks. J Power Sources 173:19-27. doi:10.1016/j.jpowsour.2007.03.055

13. Maranzana G, Moyne C, Dillet J, Didierjean S, Lottin O (2010) About internal currents during start-up in proton exchange membrane fuel cell. J Power Sources 195:5990-5995. doi:10. 1016/j.jpowsour.2009.10.093

14. S++ Simulation Services, Gräfin-Justitia-Straße 2, 82544 Egling/Thanning, Germany. www.splusplus.com

15. Candusso D, Poirot-Crouvezier JP, Bador B, Rullière E, Soulier R, Voyant JY (2004) Determination of current density distribution in proton exchange membrane fuel cells. Eur Phys J Appl Phys 25:67-74. doi:10.1051/epjap:2003079

16. Sailler S, Rosini S, Chaib MA, Voyant JY, Bultel Y, Druart F, Ozil P (2007) Electrical and thermal investigation of a selfbreathing fuel cell. J Appl Electrochem 37:161-171. doi:10.1007/ s10800-006-9229-5

17. Hauer KH, Potthast R, Wüster T, Stolten D (2005) Magnetotomography: a new method for analysing fuel cell performance and quality. J Power Sources 143:67-74. doi:10.1016/j.jpowsour. 2004.11.054

18. Lustfeld H, Reißel M, Steffen B (2009) Magnetotomography and electric currents in a fuel cell. Fuel Cells 4:474-481. doi:10.1002/ fuce. 200800139

19. Le Ny M, Chadebec O, Cauffet G, Dedulle JM, Bultel Y (2012) A three dimensional electrical model of PEMFC stack. Fuel Cells 12:225-238. doi:10.1002/fuce.201100101

20. Le Ny M, Chadebec O, Cauffet G, Dedulle JM, Bultel Y, Rosini S, Fourneron F, Kuo-Peng P (2013) Current distribution identification in fuel cell stacks from external magnetic field measurements. IEEE Trans Magn 49(5):1925-1928. doi:10.1109/ TMAG.2013.2239967 